\title{
Modification of a haematoxylin, eosin, and natural saffron staining method for the detection of connective tissue
}

\author{
Cassandra Ceccopieri ${ }^{\bowtie}$, Joanna Skonieczna, Jan P. Madej \\ Division of Histology and Embryology, Department of Biostructure and Animal Physiology, \\ Wrocław University of Environmental and Life Sciences, 50-375 Wrocław, Poland \\ ceccopieri@yahoo.com
}

Received: August 10, $2020 \quad$ Accepted: January 12, 2021

\begin{abstract}
Introduction: The aim of our study was to optimise an existing staining procedure: haematoxylin-eosin saffron (HES). The method follows the classical haematoxylin and eosin protocol with the addition of a staining step using natural saffron to better identify the collagen fibres. Material and Methods: The saffron solution was obtained by dissolving ground saffron stigmas in absolute alcohol. In order to test the HES method for its staining ability on four main types of collagen (I, II, III, and IV), specific tissues (skin, tooth, cartilage, aorta, spleen, and penis) were chosen. Results: The procedure showed a sharp differentiation between muscle, stained red or pink, and connective tissue, stained bright yellow or orange. Conclusion: HES allows the diagnosis of reticulin fibrosis undetected in HE and in previous saffron staining procedures. HES represents an advantageous alternative to HE staining giving highly reproducible results with high diagnostic value.
\end{abstract}

Keywords: saffron, collagen fibres, multichromatic staining, connective tissue.

\section{Introduction}

Saffron is a spice obtained from the threads of Crocus sativus' flowers. Originating in the Middle East, saffron has been used for centuries as a spice and colouring agent for food and clothes (13). The main bioactive compounds of saffron are picrocrocin, responsible for the bitter taste; safranal, emitting the scent; and the carotenoid pigments crocetin and crocin, which give the typical vivid crimson colour (1). Crocins, safranal, and picrocrocin have been proved to have a cytotoxic effect on human cancer cells (11).

In 1719 saffron was used by Leeuwenhoek to stain animal tissue sections and simplify the microscopic analysis. In the $20^{\text {th }}$ century, Conn applied it to detect liver damage and obtain differential staining for the glandular cells of the stomach (9). Saffron was then employed to distinguish collagen fibres in several multichromatic stains such as a variant of Masson's trichrome using haematin, phloxine, and saffron (3) or modified Russell-Movat pentachrome (10).

Collagen is the primary structural element of the dermis of vertebrates and it makes up to $30 \%$ of total body protein in mammals (7). It represents the main component of the extracellular matrix and is, like most of the extracellular fibres, an acidophilic structure (6). The acidity of its water-soluble carotenoids makes saffron a suitable dye for collagen fibres.

The function of collagen is to confer strength and support for the organs. It can be found in many parts of the body and occurs in different types, of which almost 30 are known (7). The most frequently found are the fibrillar types (I, II, III, V, XI, XXIV, and XXVII) that are characterised by extensive cross-linking which provides mechanical strength for high-stress tissues like skin, cartilage, and bone (5).

When secreted in excess, collagen may lead to tissue hardening and overgrowth causing a pathological change called fibrosis. Fibrosis is usually a side effect of chronic inflammatory reactions triggered by different kinds of stimuli like physical or chemical insults, infections, hypersensitivity reactions, autoimmune responses, and radiation (14).

In 1997 Edston and Gröntoft (4) introduced the first method adding a saffron staining step to the classical haematoxylin and eosin substitute erythrosin B protocol. The procedure gives impressive results in differentiating the dermis from the muscular tissue underneath it in skin 
samples, but it fails to clearly distinguish muscle from connective tissue in several other organs like blood vessels, the spleen, and the penis. Furthermore, in the case of tooth samples, the aforementioned method does not stain the collagen present in the dentin.

We introduce an improved version of the method presented by Edston and Gröntoft succinctly described as haematoxylin-eosin saffron (HES). It consists of a trichromatic staining procedure for routine use in histological laboratories that facilitates the identification of collagen fibres and accelerates pathological investigation of connective tissue in a wide range of organs.

\section{Material and Methods}

Tissues. To evaluate the HES method for its ability to stain the four main types of collagen (I, II, III, and IV), skin, tooth, cartilage, aorta, spleen, and penis tissues were selected. The tissue samples (stored in paraffin blocks) were retrieved from the archive of the Division of Histology and Embryology of Wrocław University of Environmental and Life Sciences. The organs had previously been fixed in a $4 \%$ buffered formaldehyde solution, washed in running tap water, dehydrated, and cleared in xylene, suitably preparing them for use in a Shandon Citadel 1000 automatic tissue processor (Thermo Fisher, Germany) in standard mode. The embedding procedure in paraffin was performed using a Myr Ec 500 tissue embedding centre (Especialidades Médicas Myr S.L., El Vendrell, Spain). Sections of $5 \mu \mathrm{m}$ were cut with an HM 310 microtome (Microm International, Walldorf, Germany) and placed on glass slides (Thermo Fisher Scientific, Waltham, MA, USA).

Solutions. The solution of saffron in alcohol was prepared as follows. One gram of natural saffron stigmas (from a food market) was ground into a powder, dissolved in $15 \mathrm{~mL}$ of absolute alcohol, mixed and heated at $35-40^{\circ} \mathrm{C}$ for $30 \mathrm{~min}$ on a heated stirrer. The solution was then filtered and stored at room temperature for further use. To obtain the eosin staining solution, $10 \mathrm{~g}$ of eosin powder (Avantor Performance Materials, Gliwice, Poland) was dissolved in $1 \mathrm{~L}$ of distilled water and mixed for $30 \mathrm{~min}$ at $35-40^{\circ} \mathrm{C}$ on a heated stirrer. Once the solution was homogeneous, 10 drops of glacial acetic acid were added. The haematoxylin staining steps were performed using the ready-to-use solution according to Delafield (CAS no. MHS80; SigmaAldrich, Darmstadt, Germany). Masson-Goldner trichrome and Reticulin silver plating according to Gordon and Sweet's histological procedures were carried out respectively using the CAS no. 1004850001 and CAS no.1002510001 Sigma-Aldrich staining kits. Once stained, the slides were mounted using the Euparal ready-to-use microscopic mounting solution (CAS no. 7356.1; Carl Roth, Karlsruhe, Germany).
HES procedure. The cut sections were mounted on glass slides, deparaffinised in xylene, and rehydrated in a degressive series of ethanol solutions. The samples were incubated in haematoxylin solution for $4 \mathrm{~min}$, then rinsed in distilled water and immersed in acid alcohol for $1 \mathrm{~min}$. The slides were then washed in running tap water for $10 \mathrm{~min}$, incubated in eosin solution for $4 \mathrm{~min}$ and rinsed in distilled water for a few seconds. Subsequently, the specimens were briefly immersed in $96 \%$ ethanol and incubated in the saffron alcohol solution (prewarmed to $35-40^{\circ} \mathrm{C}$ for $10 \mathrm{~min}$ ) for $15 \mathrm{~min}$; the slides were examined microscopically after 5 min incubation in the saffron solution following a fast rinse in distilled water as is recommended. The slides were then immersed in two changes of absolute alcohol for $5 \mathrm{~min}$ and mounted with cover slides.

\section{Results}

The procedure sharply differentiated between muscle, stained red or pink, and connective tissue stained bright yellow or orange.

HES for routine stainings. The artery wall (mouflon aorta) slides exemplify how HES (Fig. 1B) gives improved contrast between connective tissue and muscular tissue staining colours in comparison to the haematoxylin-erythrosine saffron procedure (Fig. 1A). A similar result was obtained in chicken spleen (Fig. 1C and D), where the HES-stained sample showed a more visible contrast between reticular fibres (type III collagen) meshwork and the cells in the red and white pulp. The collagen type I-rich connective tissue of the trabeculae as well as smooth muscle cells in the artery wall are also clearly visible. In rat skin (Fig. 1E and F), the HES and haematoxylin-erythrosine saffron staining procedures gave comparable results. However, the saffron dye of HES allowed a higher saturation of the connective tissue stain to be achieved in the dermis.

In Fig. 2A, the saffron dye of the haematoxylinerythrosine saffron procedure failed to stain the connective tissue in the pig penis, which only took a dark red colour from the erythrosine B dye. In contrast, the HES-dyed specimen (Fig. 2B) showed a clear distinction between the pink colour of the muscle cells in the tunica media of the artery wall given by eosin, and the yellow colour given by saffron to the collagen of the connective tissue of the penis. Furthermore, unlike the haematoxylin-erythrosine-saffron procedure, HES stained the collagen in the basal membrane. In hyaline cartilage (Fig. 2C and D), HES and haematoxylinerythrosine saffron staining achieved very similar results but there was still higher contrast in the HES-stained sample. In rabbit tooth samples (Fig. 2E and F), haematoxylin-erythrosine saffron did not stain the dentin. Contrary findings were made with HES, which impregnated this sample satisfactorily. 
HES in diagnostic applications. HES staining is also very useful in the diagnosis of reticulin fibrosis. Both type I and III collagen are clearly visible (Fig. 3D), while the structure of the cell cytoplasm is clearer than in Masson-Goldner trichrome staining (Fig. 3B). Haematoxylin-erythrosin saffron staining (Fig. 3A) applied to the same specimen of cavy liver results in lower contrast between connective and glandular (epithelial) tissue. Type III collagen is also more difficult to discern. Similar results to those of HES were obtained only with silver impregnation, an elaborate and specific methodology designed to stain type III collagen.
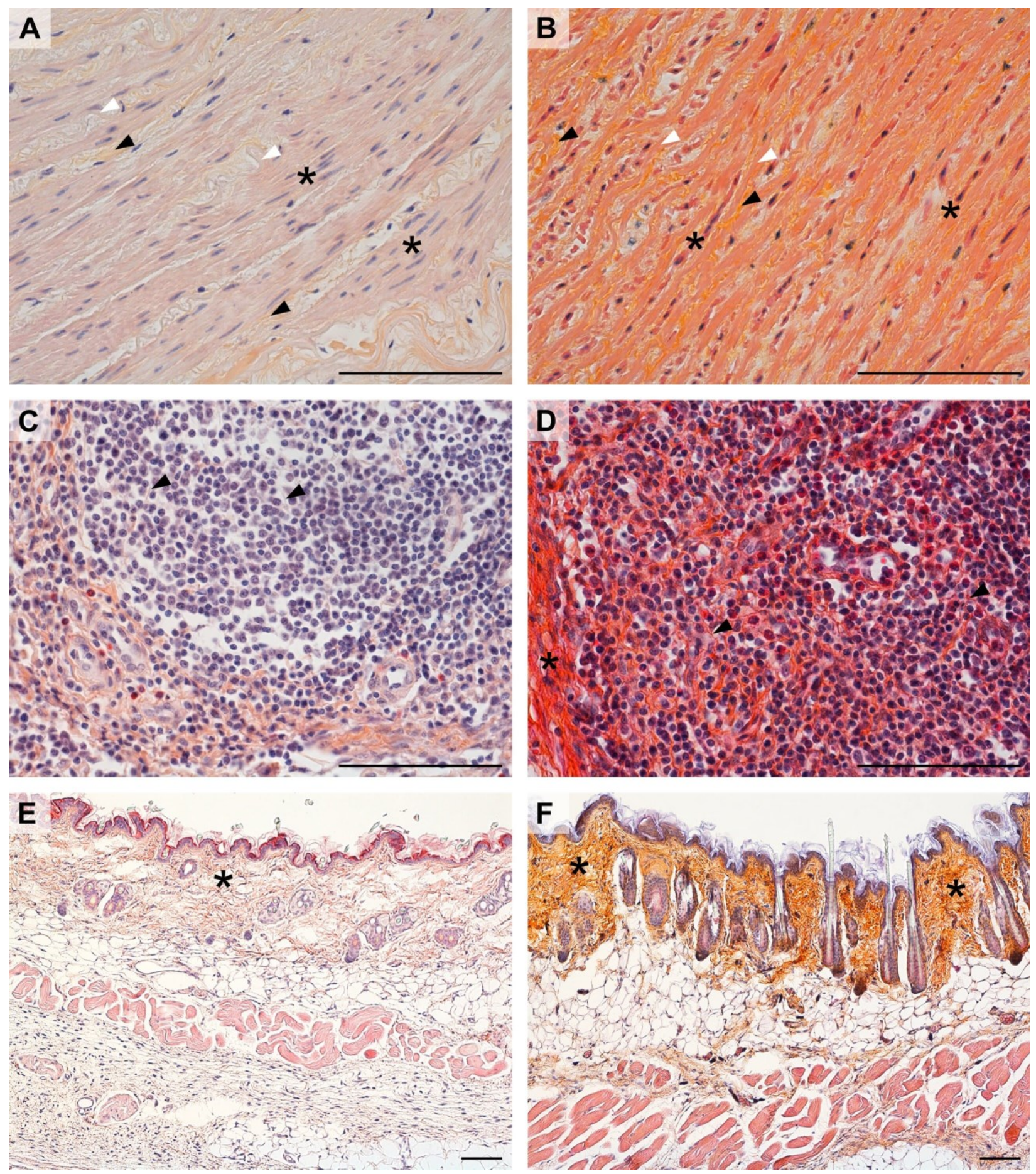

Fig. 1. Comparison of staining outcomes of the haematoxylin-erythrosine saffron method (A, C, and E) and haematoxylin-eosin saffron (HES) method $(\mathrm{B}, \mathrm{D}$, and $\mathrm{F})$

A and B - mouflon aorta; black arrowhead - collagen (mainly type I) fibres; white arrowhead - elastic fibres; asterisk - smooth muscle cells

$\mathrm{C}$ and D - chicken spleen; asterisk - trabeculae; arrowhead - reticular (type III collagen) fibres

$\mathrm{E}$ and $\mathrm{F}$ - rat skin; asterisk - collagen of the dermis. All scale bars $=100 \mu \mathrm{m}$ 

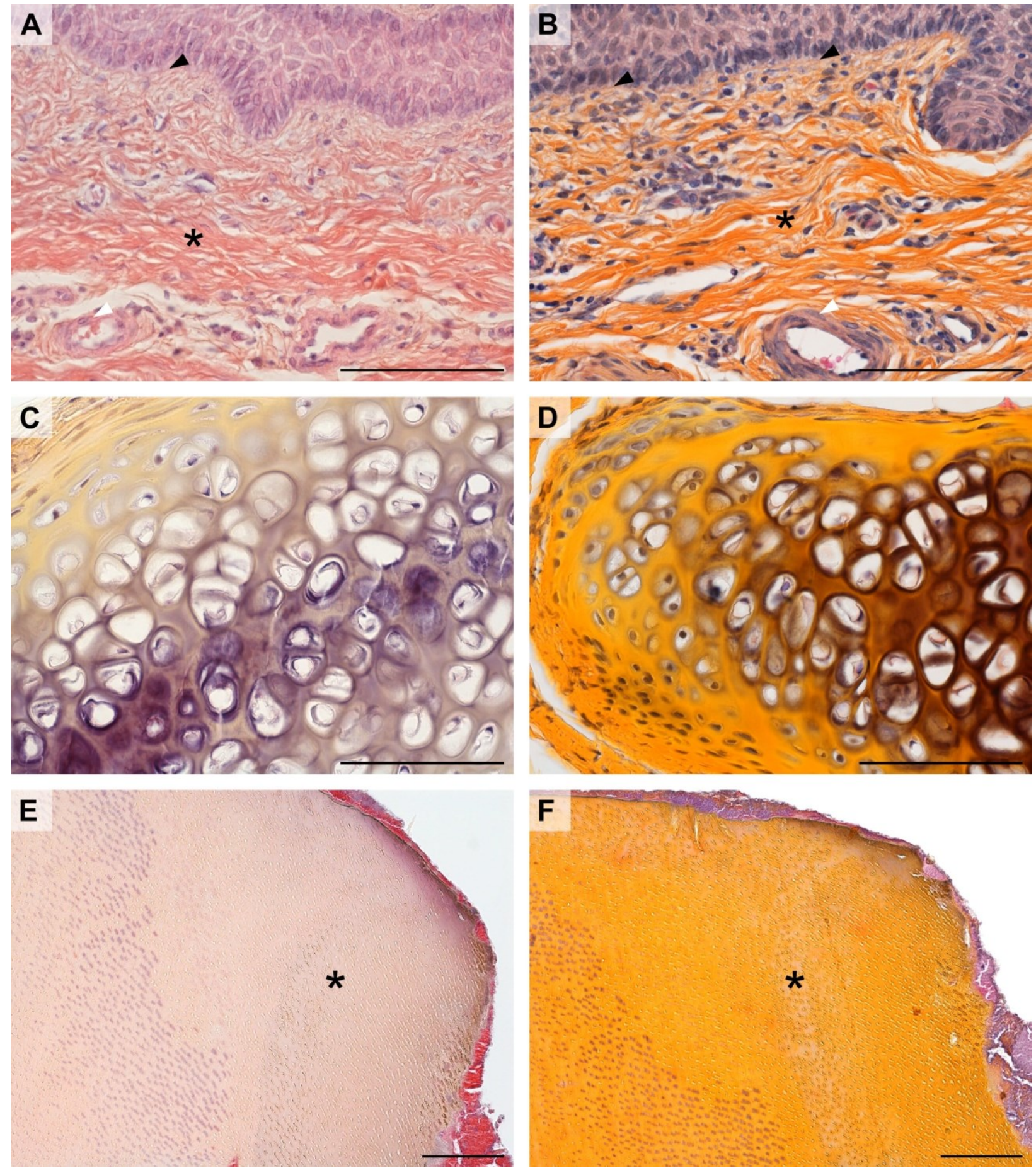

Fig. 2. Comparison of staining outcomes of the haematoxylin-erythrosine saffron method (A, C, and E) and haematoxylin-eosin saffron (HES) method $(\mathrm{B}, \mathrm{D}$, and $\mathrm{F})$

A and B - pig penis; black arrowhead - basement membrane; asterisk - collagen (mainly type I) fibres; white arrowhead - smooth muscle cells $\mathrm{C}$ and $\mathrm{D}$ - hyaline cartilage from the trachea of the rabbit

$\mathrm{E}$ and $\mathrm{F}-$ rabbit tooth; asterisk - dentin. All scale bars $=100 \mu \mathrm{m}$

\section{Discussion}

The procedure described in this article maintains the characteristics of the HE protocol with the advantage of giving a clear distinction between collagen and muscle.

The specificity of interaction with collagen fibres is probably due to the chemical nature of the stain. Indeed, when a combination of large and small acid dyes is applied, the largest and slowest diffusing dyes, like saffron, tend to accumulate first in the more hydrated elements of the tissues with great porosity and highly accessible surface area like collagen fibres. Contrastingly, small acid dyes establish nonspecific (most probably electrostatic) interactions with the amine group of amino acids: for instance, eosin binds to free $\mathrm{NH}_{2}$ groups in proteins (2). 

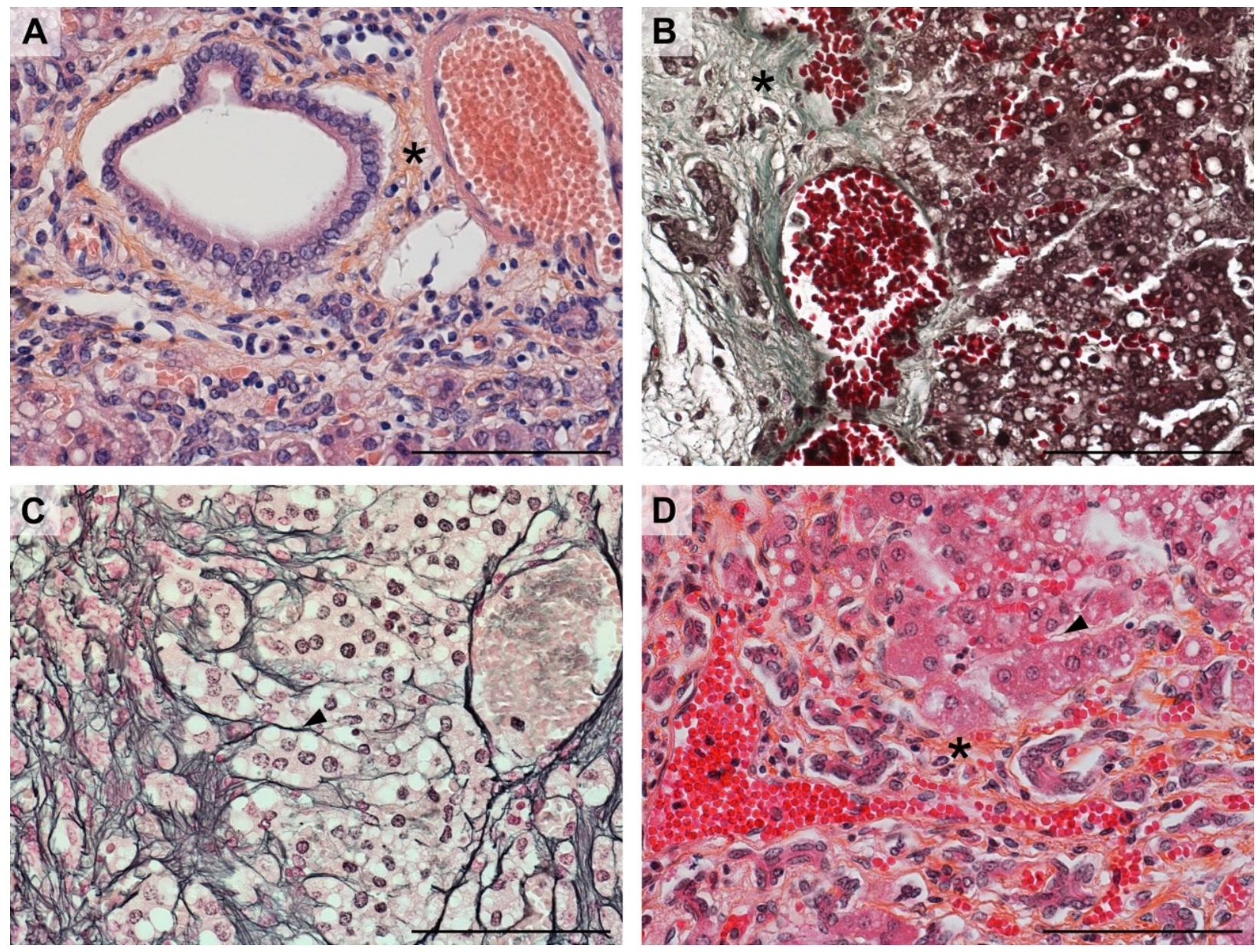

Fig. 3. Comparison of different staining methods in the diagnosis of reticulin fibrosis in cavy liver

A - haematoxylin-erythrosine saffron method

B - Masson-Goldner trichrome

C - silver impregnation

D - haematoxylin-eosin saffron (HES) method; asterisk - collagen (mainly type I) fibres; arrowhead - reticular (type III collagen) fibres. All scale bars $=100 \mu \mathrm{m}$

When compared with previous methods employing saffron (4), HES shows a wider spectrum of application allowing the collagen fibres to be distinguished from the surrounding tissues with high contrast in a great variety of organ samples. Furthermore, saffron represents a non-toxic alternative to other substances. Indeed, several multichromatic stains employ dyes like metanil yellow, a $\mathrm{pH}$ indicator known for its neuro- (8) and hepatotoxicity (12) as a food colourant also classified as a corrosive and chronic hazard to aquatic life (Metanil Yellow, PubChem Database, CID 393558, contained for example Alcian Blue-HE Metanil Yellow stain). Other methods, like Reticulin silver plating according to Gordon and Sweet, apply corrosive silver nitrate (PubChem Database, CID 24470), and formalin, a carcinogenic agent (formaldehyde, PubChem Database CID 712), to detect collagen (type III) presence in tissue.

HES offers improved diagnostic utility, as clearly shown in the liver sample, where saffron allowed fibrosis of the reticular fibres to be revealed. Whereas fibrosis was not detected by the HE or haematoxylin- erythrosin saffron methods. Similar results were obtained only with silver impregnation, an elaborate procedure that requires the employment of more toxic substances.

Therefore, HES method represents a great alternative to HE staining giving highly reproducible results with high diagnostic value. This method is also easy and rapid to perform and requires less toxic and expensive chemicals than other techniques. Moreover, the interpretation of the results of the HES method is straightforward because of its analogy to those of classical HE.

Conflict of Interests Statement: The authors declare that there is no conflict of interests regarding the publication of this article.

Financial Disclosure Statement: The publication of the article was financed under the Leading Research Groups' support project from the subsidy for the period 2020-2025 in the amount of $2 \%$ of the subsidy referred to Art. 387 (3) of the Law of 20 July 2018 on Higher Education and Science, obtained in 2019. 
Animal Rights Statement: Not applicable.

\section{References}

1. Amanpour A., Kelebek H., Selli S.: GLC/HPLC Methods for Saffron (Crocus sativus L.). In: Bioactive Molecules in Food, edited by J.M. Mérillon, K.G. Ramawat, Springer, Cham, 2019, pp. 1987-2035.

2. Bathaie S.Z., Farajzade A., Hoshyar R.: A review of the chemistry and uses of crocins and crocetin, the carotenoid natural dyes in saffron, with particular emphasis on applications as colorants including their use as biological stains. Biotech Histochem 2014, 89, 401-411, doi: 10.3109/10520295.2014.890741.

3. Bencosme S.A.: A trichrome staining method for routine use. Am J Clin Pathol 1954, 24, 1324-1328, doi: 10.1093/ajcp/ 24.11_ts.1324.

4. Edston E., Gröntoft L.: Saffron - A connective tissue counterstain in routine pathology. J Histotechnol 1997, 20, 123-125, doi: 10.1179/his.1997.20.2.123.

5. Firestein G., Budd R., Gabriel S.E., McInnes I.B., O’Dell J.: Heritable Diseases of Connective Tissue. In: Kelley and Firestein's Textbook of Rheumatology 10th edition, edited by G.S. Firestein, R.C. Budd, S.E. Gabriel, I.B. McInnes, J.R. O’Dell, Elsevier, Philadelphia, 2016, pp.1797-1815.

6. Mescher A.L.: Junqueira's Basic Histology: Text \& Atlas, 15th edition edited by M. Weitz, B. Kearns, P. Boyle, McGraw-Hill Medical, New York, 2018, pp. 96-103.
7. Mienaltowski M.J., Birk D.E.: Structure, physiology, and biochemistry of collagens. Adv Exp Med Biol 2014, 802, 5-29, doi: 10.1007/978-94-007-7893-1_2.

8. Nagaraja T.N., Desiraju T.: Effects of chronic consumption of metanil yellow by developing and adult rats on brain regional levels of noradrenaline, dopamine and serotonin, on acetylcholine esterase activity and on operant conditioning. Food Chem Toxicol 1993, 31, 41-44, doi: 10.1016/0278-6915(93)90177-z.

9. Pearse A.G.E.: History of staining 3rd edition, edited by G. Clark, F.H. Kasten, Williams and Wilkins, Baltimore 1983, p. 139.

10. Russell H.K. Jr.: A modification of Movat's pentachrome stain. Arch Pathol 1972, 94, 187-191.

11. Samarghandian S., Borji A.: Anticarcinogenic effect of saffron (Crocus sativus L.) and its ingredients. Pharmacognosy Res 2014 6, 99-107, doi: 10.4103/0974-8490.128963.

12. Saxena B., Sharma S.: Food Color Induced Hepatotoxicity in Swiss Albino Rats, Rattus norvegicus. Toxicol Int 2015, 22 , 152-157, doi: 10.4103/0971-6580.172286.

13. Schmidt T., Heitkam T., Liedtke S., Schubert V., Menzel G.: Adding color to a century-old enigma: multi-color chromosome identification unravels the autotriploid nature of saffron (Crocus sativus) as a hybrid of wild Crocus cartwrightianus cytotypes. New Phytol 2019, 222, 1965-1980, doi: 10.1111/nph.15715.

14. Wynn T.A.: Cellular and molecular mechanisms of fibrosis. J Pathol 2008, 214, 199-210, doi: 10.1002/path.2277. 